\title{
FORCED EXPIRATORY VOLUME BEFORE AND AFTER ISOPRENALINE
}

\author{
BY \\ K. M. HUME AND BRYAN GANDEVIA* \\ From the Respiratory Department, King Edward VII Sanatorium, Midhurst, Sussex
}

(RECEIVED FOR PUBLICATION JANUARY 12, 1957)

Improvement in the results of tests of ventilatory function in response to a bronchodilator drug, given by inhalation or by injection, is now widely used to indicate the presence of reversible " bronchospasm" (Rossier, 1949; Gilson and Hugh-Jones, 1955; Kennedy and Thursby-Pelham, 1956). Frequent repetition of some of these tests in a small series of patients has revealed an interesting relationship between the initial values for the tests and the changes observed after the inhalation of isoprenaline.

\section{Method}

Six patients admitted to King Edward VII Sanatorium because of a severe exacerbation of " intrinsic" asthma were selected for study. It was hoped that frequent observations of a small group might prove more rewarding than isolated tests of a larger series. The diagnosis was based upon the history of recurrent attacks of severe wheezing, sometimes precipitated by a "cold," but otherwise without obvious external cause, with intervening periods of relative freedom. Except in one patient who had been asthmatic since childhood, the symptoms began during middle life. None of the patients was regarded as having predominantly chronic bronchitis. The ages of the patients ranged from 39 to 60 years; four were women.

The spirometer was of the type described by Bernstein, D'Silva, and Mendel (1952), and the spirograms were recorded on a kymograph with a peripheral drum speed of $1.7 \mathrm{~cm}$. per second. The patient was instructed to inhale as deeply as possible from the spirometer, and then to breathe out into it rapidly, and as completely as possible. Three recordings were obtained. The patient then inhaled a mist of $1 \%$ isoprenaline in saline from a Collison inhaler for one minute, about $0.5 \mathrm{ml}$. of the liquid being nebulized in this time. Previous experiments, as well as repeated observations in the course of the present study, showed that this method and duration of administration are adequate to produce the greatest possible effect. Two minutes after the inhalation three further spirograms were recorded in the same way as before. The volume of air expired in the first $0.75 \mathrm{sec}$., $1.0 \mathrm{sec}$., and $2.0 \mathrm{sec}$. of each maximal expiration was estimated from the spirograms; each of these volumes is referred to as the forced expiratory volume at the stated

* Wunderly Scholar in Diseases of the Chest, Royal Australasian College of Physicians.

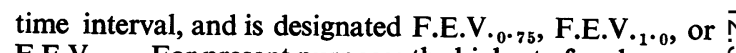
F.E.V. $\cdot_{\cdot 2 \cdot 0}$. For present purposes the highest of each group of three recordings was selected. The total volume of air expired (the forced vital capacity) was also noted. The tests were repeated before and after isoprenaline every day while the patient was in hospital, and thereafter weekly.

During the investigation, treatment with prednisone acetate ( $25 \mathrm{mg}$. daily) was begun in five of the patients; reduction in dosage (by $5 \mathrm{mg}$. every three days) was begun when the daily fast expiratory capacity values ceased to show further improvement. In view of the recognized liability to relapse in these cases, the oral administration of choline theophyllinate $(200 \mathrm{mg}$. three or four times daily) was begun at this stage; this was continued afte 8 prednisone had been stopped. The remaining patien was treated throughout with choline theophyllinate alone.

\section{RESULTS}

The effect of isoprenaline was observed on 150 occasions. The initial values for the F.E.V. ${ }_{75}$, F.E.V.1 ${ }_{0}$, and F.E.V.2.0 against the respective increases recorded after the inhalation of isoprenaline. The 18 curves thus obtained were strikingly similar in shape (Fig. 1), with a well-defined maximum value for the increase after isoprenaline, corresponding to a value between the lowest and highest recorded for the pre-inhalation forced expiratory volume. In all cases this peak was observed after five to seven days of treatment. This relationship is not merely a function of improvement in response with the passage of time, as can be shown by re-plotting the data with duration of hospital stay as abscissa.

Three curves for the F.E.V.1.0 are reproduced (Fig. 1). Patient A was studied through two complete cycles of exacerbation and remission; the results were so similar that they are here shown as a single curve.

When the results for the total amount of air expired (forced vital capacity) were similarly plotted, the shape of the curves was found to be the same. However, the points showed a much greater scatter, related in some instances to marked differences in the time taken over expiration before 


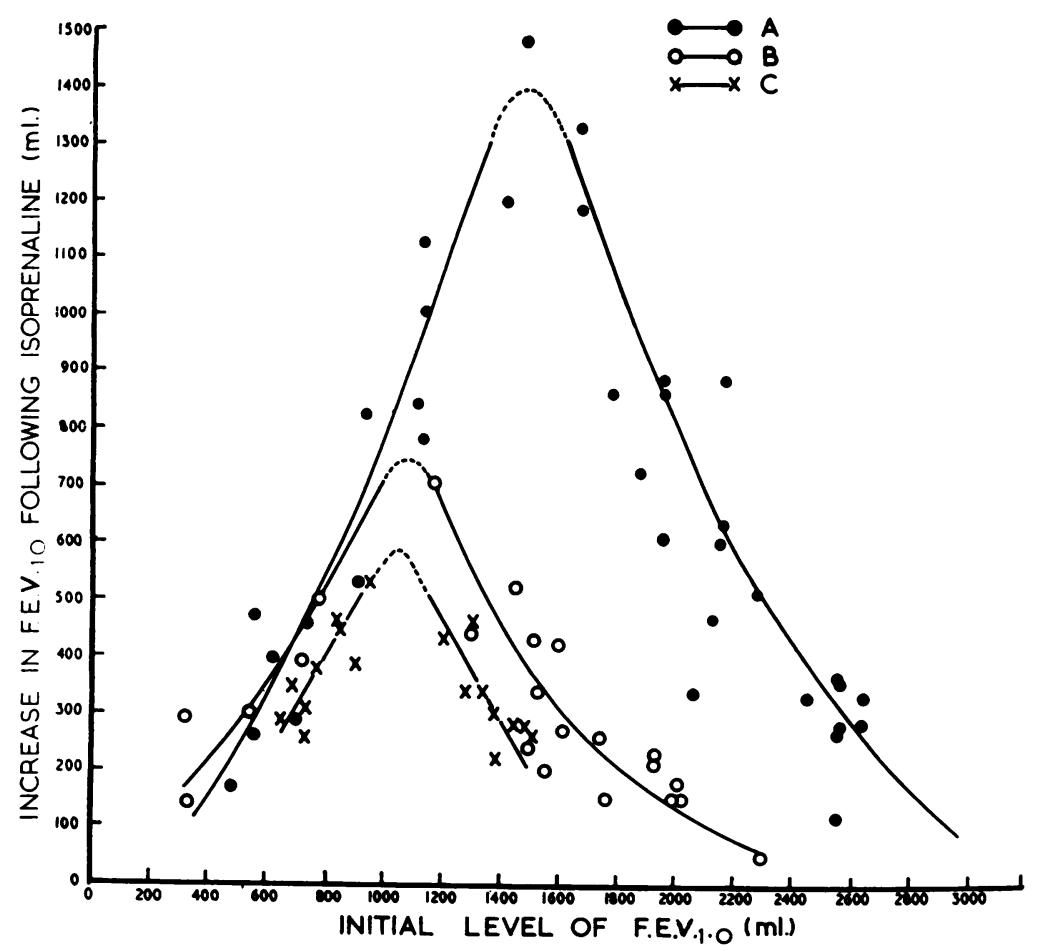

FIG. 1.-The relationship between the initial level of forced expiratory volume in the first second and the increase in this volume after isoprenaline inhalation, plotted for three patients. Patient A was studied during two distinct exacerbations and remissions, but both series of observations may be represented by a single curve.

and after the administration of isoprenaline. This was exemplified in one patient where the relationship, in terms of F.V.C., was in fact better represented by a linear than a quadratic equation; three large increases achieved during greatly prolonged expirations were included in the early observations in this case. It may well be that more reliable and consistent results would be obtained by estimating vital capacity during a slow expiration.

In one of the six patients several of the earlier observations showed a much smaller response than the curve tentatively plotted from some subsequent results would have suggested. The reason for this is unknown.

The patient was a formerly athletic man aged 60 years who had a six months' history of asthmatic attacks. He had had no illness before this. On clinical and radiological grounds it was thought that he might also have had emphysema. He was the only patient in the series producing considerable quantities of sputum. At first he coughed up bronchial casts, but during the period of apparent " isoprenaline resistance" the sputum was thick, glairy, and colourless, about $2 \mathrm{oz}$. $(60 \mathrm{ml}$.) per day being produced. The tests evoked violent coughing, but the mucus was brought up with the utmost difficulty.
The small quantities produced after each bout of coughing rapidly formed a homogeneous mass in the sputum mug. It is also notable that even when this patient was remarkably free of respiratory symptoms, during prednisone therapy, he had a vital capacity of only $2,400 \mathrm{ml}$. (about two-thirds his predicted value), and he could expire only $50 \%$ of this within the first second (normal, $75-85 \%$ ). It is possible that the comparatively unsatisfactory curve obtained in this patient was related to the excessive production of a peculiarly viscid mucus in conjunction with permanent structural changes in the lungs.

From the curves shown in Fig. 1, it can be seen that, to the left of the peak, the response increases with increase in the pre-inhalation level of the F.E.V.1.0. Over the entire series this increase ranges from about $30 \%$ to $90 \%$ of the initial level, and it is not necessarily constant for any individual. To the right of the peak, the sum of the initial level and the increase after isoprenaline remains approximately constant, the percentage increase falling. The relative constancy of the post-inhalation F.E.V. ${ }_{10}$ over this range means that in theory much of this limb of the curve could be plotted from a knowledge of any one point past the peak. Similar comments apply to the curves based upon the values for the fast vital capacity. 


\section{Discussion}

The results indicate that, as the forced expiratory volume and vital capacity improve in severely ill patients, so does the absolute response to inhaled isoprenaline; at a certain level this response reaches a maximum. As further improvement occurs, the isoprenaline response diminishes progressively, reaching a minimum when the maximum forced expiratory or vital capacity has been attained. It may be pointed out that in order to demonstrate this pattern of response it is necessary to study a patient over a wide range of initial forced expiratory volume or vital capacity values. Whether the relationship occurs in all cases of reversible bronchial obstruction remains to be seen; in only one of our patients were there several points which clearly did not fit the expected curve.

The absolute response to isoprenaline inhalation is relatively poor when the initial forced expiratory values are low. The reason for this is not certain. The shape of the curves plotted in Fig. 1 strongly suggests that at least two factors are involved in the bronchodilator effect of isoprenaline; these may be related to two or more of the recognized factors in asthmatic wheezing, namely, bronchial spasm, mucosal oedema or inflammation, and mucus plugging. The lack of progressive improvement when the time of isoprenaline administration is prolonged suggests that the relatively small response is not due simply to ineffective ventilation, or at least to ineffective ventilation of those parts of the lung capable of any ventilation at all. This observation is supported clinically by auscultation of the chest in some patients during the inhalation of isoprenaline. The characteristic high-pitched rhonchi present at rest disappear after two or three breaths of isoprenaline mist. At this stage highpitched rhonchi can still be elicited by forced expiration, and these are unaltered, as far as can be ascertained, by further inhalation of isoprenaline.

The implications of this relationship between response to isoprenaline and the initial level of forced expiratory volume and vital capacity are of interest. Extrapolation of the right-hand limb of the curve relating response to initial level offers an approximate estimate of the maximum value which a particular patient may be expected to attain. Comparison of this figure with a value predicted from the age, sex, and stature of the patient may then provide an estimate of the lung damage present which is irreversible by the therapy employed. Thus, from the "isoprenaline response curve" A in Fig. 1 it can be seen that the highest likely F.E.V..$_{10}$ is about $3,000 \mathrm{ml}$. From the similar curve constructed for forced vital capacity in this patient, the maximum vital capacity was found to be $4,500 \mathrm{ml}$., which was the same as the predicted normal for this patient. As he can expire approximately $66 \%$ (normal $75-85 \%$ ) of this within the first second, he may be regarded as having relatively little irreversible lung damage. On the other hand, one of the patients could expire no more than $50 \%$ of a rather low vital capacity, even at her best, and in the virtual absence of demonstrable reversible bronchial constriction.

Construction of an " isoprenaline response curve" of the type described may prove helpful in controlling cortisone or prednisone dosage, particularly when it is desired to keep this to a minimum. None of the patients was conscious of respiratory disability when the forced expiratory volume was at the level associated with the maximum isoprenaline response. The maintenance dosage selected may therefore be kept to that necessary to maintain this level, rather than that required to achieve a more " normal" state.

Finally, it is possible that the pattern of response to isoprenaline depicted in the graphs may be found to apply to other bronchodilator drugs. If this should prove to be the case, care must be taken in evaluating the results of ventilatory function tests when they are used to compare the effects of oneon drug with another. In prolonged clinical trials there may be not only the well-recognized hazard of spontaneous variation in the initial level of fast expiratory capacity, but also a risk of concomitant variation in the response produced by the drugs under trial. It may be difficult to make allowance for this effect.

\section{SUMMARY}

Estimations of forced expiratory volume have been carried out at frequent intervals in six patients with intrinsic asthma during exacerbations and remissions. A relationship is demonstrated between the forced expiratory volume measured over three time intervals and the increase following the inhalation of isoprenaline. The implications of this relationship are discussed.

Our thanks are due to Sir Geoffrey Todd, K.C.V.O., O.B.E., and to Dr. F. J. Prime for their advice and assistance in the preparation of this paper. Supplies of prednisone acetate and of choline theophyllinate were kindly made available by Glaxo Laboratories and Messrs. Allen and Hanburys, Ltd., respectively.

\section{REFERENCES}

Bernstein, L. D'Silva, J. L and Mendel, D. (1952). Thorax, 7, 255. Bernstein, L., D'Silva, J. L., and Mendel, D. (1952). Thorax, 7, 255.
Gilson, J. C., and Hugh-Jones, P. (1955). Lung Function in Coal-
workers' Pneumoconiosis. workers' Pneumoconiosis. Spec. Rep. Ser. med. Res. Coun. (Lond.), No. 290. Kennedy, M. C. S., and Thursby-Pelham, D. C. (1956). Brit. med.

Rossier, P. H. (1949). Rev. méd. Suisse rom., 69, 686. 